\title{
VIRTUAL LABORATORY FOR COMPUTER-AIDED DESIGN OF BIOSENSORS
}

\author{
O. Palagin, V. Romanov, I. Galelyuka, M. Kachanovska \\ V.M. Glushkov Institute of Cybernetics of National Academy of Sciences of Ukraine, \\ dept230@insyg.kiev.ua, galib@gala.net
}

\begin{abstract}
Fast development of information technologies puts strict requirements to terms and costs of designing of computer devices and systems. These requirements may be fulfilled with help of virtual methods of designing, which are realized by means of virtual laboratories of computer-aided design.
\end{abstract}

Keywords: computer-aided design, CAD, biosensor, virtual laboratory, portable device, reliability.

\section{INTRODUCTION}

World and Ukrainian markets quickly are being filled with portable devices and computer facilities, which may be used in everyday life and in laboratory conditions. As examples, there are portable tonometers, cardiographs, thermometers, glucometers, gas analyzers, devices for measure of quality of foodstuff, water and environment parameters etc. Fast rise of such devices quantity causes appearance of competition between them and that is, undoubtedly, very positive feature.

Detailed analysis of portable devices market shows, in our opinion, that there is a negative tendency - almost all portable devices and computer facilities on the Ukrainian market are manufactured abroad. This situation has a place even in conditions, that a large amount of Ukrainian scientific institutes and organizations of different property forms work in the field of sensor developing, which are based on different effects and are intended for using in different devices. Detailed analysis of implement of Presidium Program of National Academy of Sciences (NAS) of Ukraine "Researches in the field of sensor systems and technologies" and results, which are published in the proceedings of conferences from biosensoric (in particular, "Sensor electronics and microsystem technologies" etc) over 2005-2007 years shows, that most of scientificresearch projects of sensors are finished by experimental sensors, most of which are not used anywhere in the future. In the mentioned materials there are dates, which show possibility to create devices for different areas and on the base of different principles of operation.

To change such situation we refer next:
1) Absence of strong relations between scientific-research organizations, which develop sensors, and specialists in circuit technology and instrument engineering, who are able to create devices on the base of these sensors. Sensor developers don't have enough information about new technologies of creating new devices on the base of their sensors, about approximate cost of devise design and production of test party of devices, and also about facilities of contract production of circuit boards, which is in Ukraine for several last years.

2) Large price of $R \& D$ stage for sensor devices in Ukraine does not permit to order test party of devices to specialists in circuit technology and instrument engineering.

Creating of special hardware-software complex may solve the problem. By means of this complex sensor developer may check possibility of device creating on basis of developed sensor, make prior calculations and estimations of device parameters (for example, cost, performance, size, reliability etc), and in some cases estimate economic benefit from project realization.

Now on the world and Ukrainian markets there are a lot of software, which allow to automotive design of new devices and systems. But for skilled usage of such CAD software it is necessary to have special skills in circuit technology, electronics and instrument engineering, and also know this CAD software perfectly. It is clear, that sensor developers, who are mainly chemists, biologists, biochemists, physicists etc, have no enough possibility and skills to use such complicated CAD software for designing 
of new devices on basis of developed sensors. In such case they need help of CAD specialists. But it is very expensive service. Therefore in most cases sensor developer leave sensor "in quiet" and switch his attention to another tasks.

Good solution of this problem is to create tools, which in convenient mode (for example, with help of dialogues) allow sensor developer to check possibility of creating of new devices and model of the device, including prior parameters calculations, project of circuit board and set of design documentations. Description of sensor or its model should be enter data for such system of design.

It is easily to see, that most devices have the same structure, to be exact, they consist of sensor, measuring channel and additional subsystems. That's why process of design could be easily formalized.

Mentioned tools allow sensor developer to:

1) Check possibility of creating of devices and computer facilities (including portable devices) on basis of developed sensors without involving specialists in circuit technology and instrument engineering at the stage of pilot-project. It allows reducing terms and costs on this stage.

2) Avoid expensive actual tests on the stage of device creating by replacing with virtual methods of designing and testing.

3) Prepare set of design documentations on designed device in the automotive mode without involving corresponding specialists. Nest stage is to send design documentations to contract production for creating of test party of devices.

For creating of such tools in V.M. Glushkov Institute of Cybernetics of NAS of Ukraine the conception and the ontology of virtual laboratory of computer-aided design (VLCAD) were developed, and later it was began creating of the virtual laboratory.

\section{VLCAD DESCRIPTION}

VLCAD, which being creating in Institute of Cybernetics, can be used not only for designing and development of certain biosensor devices, but also for estimation, testing of work hypotheses, experimental researches and training. VLCAD is worth to be used on the stage of the requirements specification or pilot-project, because it gives the possibility enough fast to estimate the project realization, certain characteristics and, as a result, expected benefit of its practical realization. On the stage of design VLCAD lets to decrease both time and cost of the design.
Offered conception of VLCAD and the virtual laboratory are created on the base of formalized representation of theoretic knowledge, principles of organization, methods and facilities of computeraided design and testing information-measuring systems and devices [1]. By formalized representation we mean computer ontology of mentioned subject field (in its extended and narrow types), which architecturally looks as hierarchical (like tree) system, which includes base essences (concepts) linked by system of relationship (genus species, element - system, cause - result etc.) with full statement of contents (interpretation function) both concepts, and their relationships. Thus VLCAD is built as ontology-driven information system with extended possibilities of intellectual and information interconnection with remote user.

Virtual laboratory is open systems and has next advantages:

1) cheapness of design, because the expensive and complicated equipment is not used and expensive actual tests are not fulfilled;

2) higher speed of design, because samples and models are already prepared before;

3) possibility of creating several alternative variants of device at the same time and choosing optimal variant from them on basis of certain criterion (for example, cost, performance, size, reliability etc);

4) possibility of working of geographicallydistributed specialists around one project. The possibility of exchange of experience between specialists is very important and useful in modern time;

5) possibility of using VLCADs for training future specialists;

6) possibility of using of databases and calculation units for scientific research;

7) possibility of creating and filling bases of knowledge and databases in the process of designing and training.

VLCAD and its simplified representation are described in [2] in detail.

Virtual laboratory is created as ontology-driven open information system with possibility of future adding new units and facilities to its structure.

VLCAD is developed using web-oriented and grid technologies. Access to virtual laboratory can be made from:

- local computer, on which the virtual laboratory is installed. The computer works as server, and as local work place;

- local network of organization (there must be server in the network);

- Internet (there must be server).

For computer-aided design of IC of NAS of Ukraine is developed using web-oriented 
technologies. Main software is allocated on the server. User software is represented by usual internet-browser, by means of which a user can access the virtual laboratory. Server software consists of user interface programs, calculation modules, databases, interactive developing tools etc. Structurally server software is divided into databases and modules. Each separated module is intended for execution of one or several related functions (for example, calculation of reliability parameters, microelectronic element database search according to certain criteria etc.). A user can interact with virtual laboratory using a common internet-browser. The user creates request using existing menus and forms. This request is delivered to server software through Internet or local network. In turn server software performs calculations by means of a certain module and sends the results to the user internetbrowser.

The typical virtual laboratory has to include the following main elements:

1) Databases consist of libraries containing all data which is necessary for virtual laboratory functioning and management. This data includes information about electronic elements, element and unit models, typical functional solutions, virtual evaluation boards, manufacturers, research objects models, interactive development tools etc. Besides, these information libraries contain models, tools and solutions.

2) Software is intended for virtual laboratory and database administration, provision of virtual research experiments and calculations, input processing, full-stage device development etc.

3) Hardware consists of technical tools intended for ensuring software operability, performing data input, storage and output.

4) Results contain some typical forms including list of device requirements, certain typical functional solutions, theoretical calculations, design documentation, requirements specifications, datasheets, application notes etc. Some of these forms are standardized, since later they can be transferred to others systems.

5) Users contain set of users typical of such systems. It includes the following ones: administrator, design engineer, customer.

6) Interfaces are intended for organization of interaction between virtual laboratory modules and interaction of virtual laboratory with users and other systems and for provision of human-machine interface.

7) Ontology-driven module represents ontology as a special kind of software for organization of the virtual laboratory operation as the ontology-driven information system.

Databases, in its turn, are divided into next libraries:

1) library of typical functional solutions, which encloses typical functional solutions for different systems, for example: data acquisition systems, data processing systems, data transferring systems, data visualization systems, diagnostic and calibration systems, power monitoring systems etc;

2) library of components, which involves expanded information about microelectronic components of different manufacturers;

3) library of microelectronic components models. This library involves SPICE and IBIS models;

4) library of evaluation boards and kids, which includes also virtual evaluation boards [3];

5) library of research object models (models of processes and effects in medicine, biology, biochemistry and other areas). This library is divided into research object models and corresponding data sets (input and output data). Library is used for realization of virtual experimental researches and providing their automation. Data sets are used for estimating of authenticity of research object models.

VLCAD software is used to perform operations on all stages of device designing, beginning from forming list of requirements to designing device and finishing by creating of design documentation, to provide normal work and administrate databases, to automate experimental researches. Thus, all software can be divided into design software, administration software and experimental research software

Design software is used on all design stages and encloses software for: forming device requirements, circuitry design, research object modeling, printed circuit boards design, device case design, device parameters calculations, creating design documentation.

Administration software is used for: providing databases integrity to make normal and uninterrupted work of system; updating libraries from global network and different data mediums; formatting data in form, which is convenient for saving in databases.

Software for experimental research is used for automation of virtual experimental researches and estimating of work hypothesis. As shown in fig. 1 this software includes software for providing experimental researches, software for forming of input data sets, software for registration of output data and software for statistical treatment of registered data. 
Hardware consists of personal computers (servers and work stations if there is distributed system), input/output facilities and facilities for information saving. If there is necessity, highly productive cluster machines will be used for tasks of multicriterion optimization, estimation of hypothesis of virtual experimental researches.

On the main page of virtual laboratory user has to perform next operations:

- getting information about virtual laboratory;

- overlooking of virtual laboratory structure in the graphical or text forms and getting information about all created units or units, which are planed to create;

- switching to the unit for calculation of reliability of microelectronic components and whole developed devices. Unit contains additional information about methods of reliability parameters calculation;

- reviewing information about microelectronic components and theirs parameters. Unit also contains data about technologies of microelectronic components production;

- reviewing library of typical functional solutions and choosing necessary variant;

- switching to the unit of interactive tools;

- switching to the library of electronic magazines, collected articles and publications;

- sending recommendations and helps to authors of VLCAD.

Interactive tools are software, which are produced by manufactures of microelectronic components or other companies, for calculation of certain parameters of microelectronic components or for providing complicated and routine calculations. Such tools are available as from Internet, so in offline mode without connection to Internet. The main reason of interactive tools creation is to lighten work of developer. As example of interactive tools there can be putted group of interactive tools "Switches and multiplexers", which are made by company Analog Devices. This group contains 4 interactive tools:

1) "Impedance matching tutorial", which examines the Smith Chart and its application to transmission line impedance matching (see fig. 1);

2) $" \mathrm{dBm} / \mathrm{dBu} / \mathrm{dBv}$ Calculator", which converts between standard units of power measurement and signal strength;

3) "Settling Time Calculator", which estimates settling time for a multiplexer by calculating the time constant of a cascaded RC network. In addition, it displays the settling time for four levels of accuracy expressed in $\% \mathrm{FS}$ and bits;
4) "Power Dissipation vs Die Temp", which estimates die temperature and power dissipation based on the supply voltages, ambient temperature, load characteristics, and package thermal data.

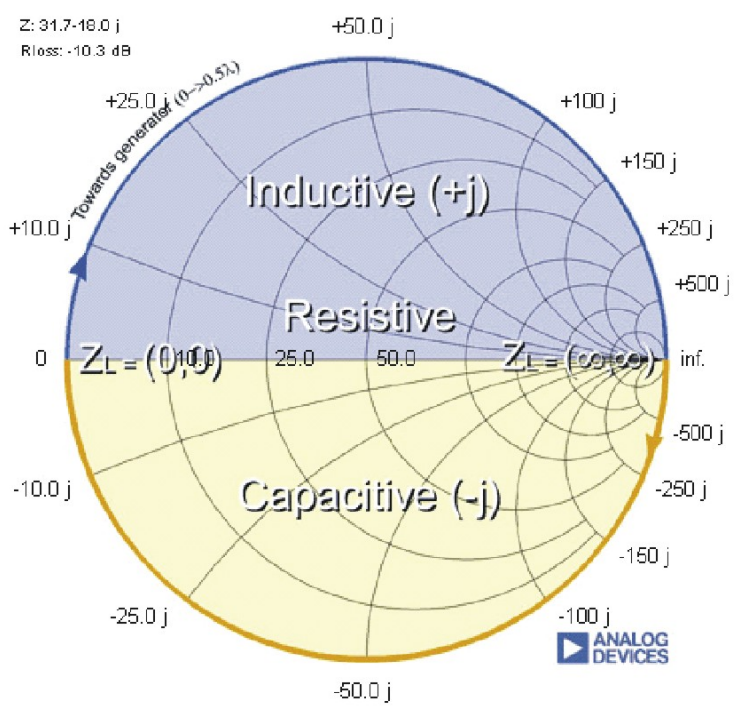

Fig. 1 - Interactive tool "Impedance matching tutorial"

\section{DESCRIPTION OF DESIGN PROCESS}

Process of device design has exact order of operations and includes next stages:

1) Forming requirements specifications of prospective device (for example, precision, efficiency, reliability, cost etc.);

2) Choosing research object model from library or creating new model (if it is absent) and testing its authenticity by means of sets of etalon data, analytical formulas etc.;

3) Circuitry designing of device on the basis of defined requirements;

4) Estimating of operating functions of the designed device and correctness of its work;

5) Previous calculating of designed device parameters (for example, precision, efficiency, reliability, cost etc.) and estimating calculated parameters for their compliance to defined requirements;

6) Designing of printed circuit boards and device cases;

7) Realizing (virtual) experimental research, tests; data registration and processing;

8) Manufacturing of evaluation board for actual testing of designed device;

9) Creating design documentation of developed device.

If the results of some stage are negative one has opportunity to return to one of previous stages. So both whole design process and every stage are iterated. 


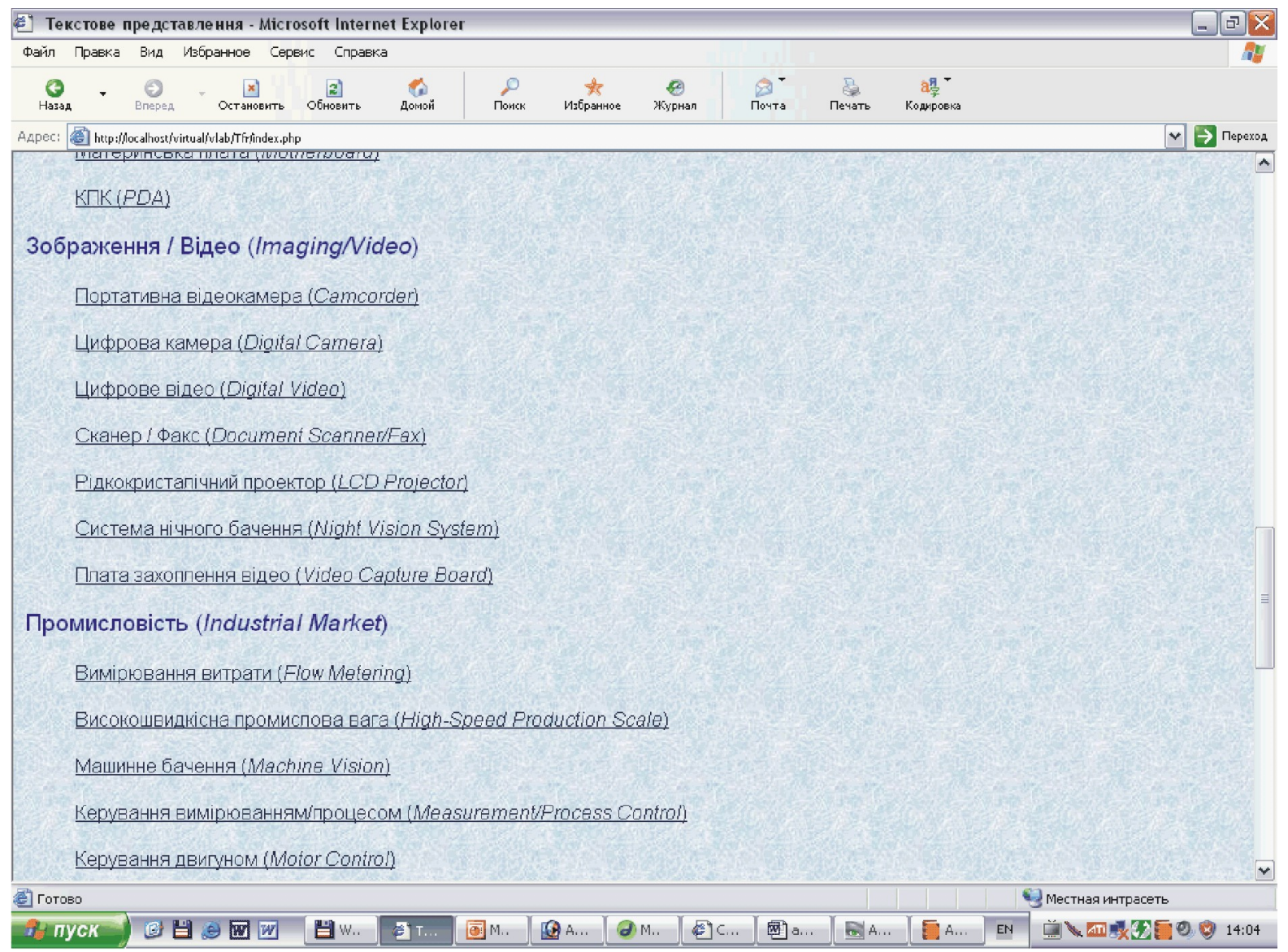

Fig. 2 - Window of library of typical functional solutions

Before designing it is possible to select example of typical functional solution from certain library. The library is divided on groups to simplify such choosing (see fig. 2.). Selected typical functional solution is shown as example on the fig. 3. Every item on the structure chart contains certain list of microelectronic components.

After selecting of the typical functional solution and list of microelectronic components one may calculates device parameters, which having been designed. To such parameters it may be referred reliability, performance, cost, error of measurement etc.

Except this parameters one may also calculates ratio of this parameters (for example, error/ reliability, error/cost, reliability/cost etc). By means of optimization the necessary ratio of parameters can be achieved.

As example, the order of automotive calculations of reliability can be shown in the next topic [4].

\section{CALCULATION OF RELIABILITY}

Calculation of reliability parameters of microelectronic components and whole devices can be made by means of two methods. First of them is widespread method on basis of exponential distribution law of fault probability (method is used by such companies, as Analog Devices, Motorola etc according to MIL-STD 883 of USA). Second method is based on DN-distribution law of fault probability and is original work of Ukrainian scientists [5]. The first distribution law is oneparameter function, the second - is two-parameters function. The order of calculation of reliability parameters by means of these two methods are the same and consist of next operations:

- define number of microelectronic components types and total number of microelectronic components in device;

- select name of every microelectronic components type from list and define number of microelectronic components on every type;

- check accuracy of entered information;

- press button "Calculate" for calculate reliability parameters of device;

- laboratory make calculations by mean of two methods for every type of microelectronic components and whole device and results will be shown on display (fig. 4).

To make calculation user may select parameters of microelectronic components from special database of VLCAD or enter necessary data manually. Parameters of microelectronic components, which are using for calculations, are taken from official sites of manufacturers. 


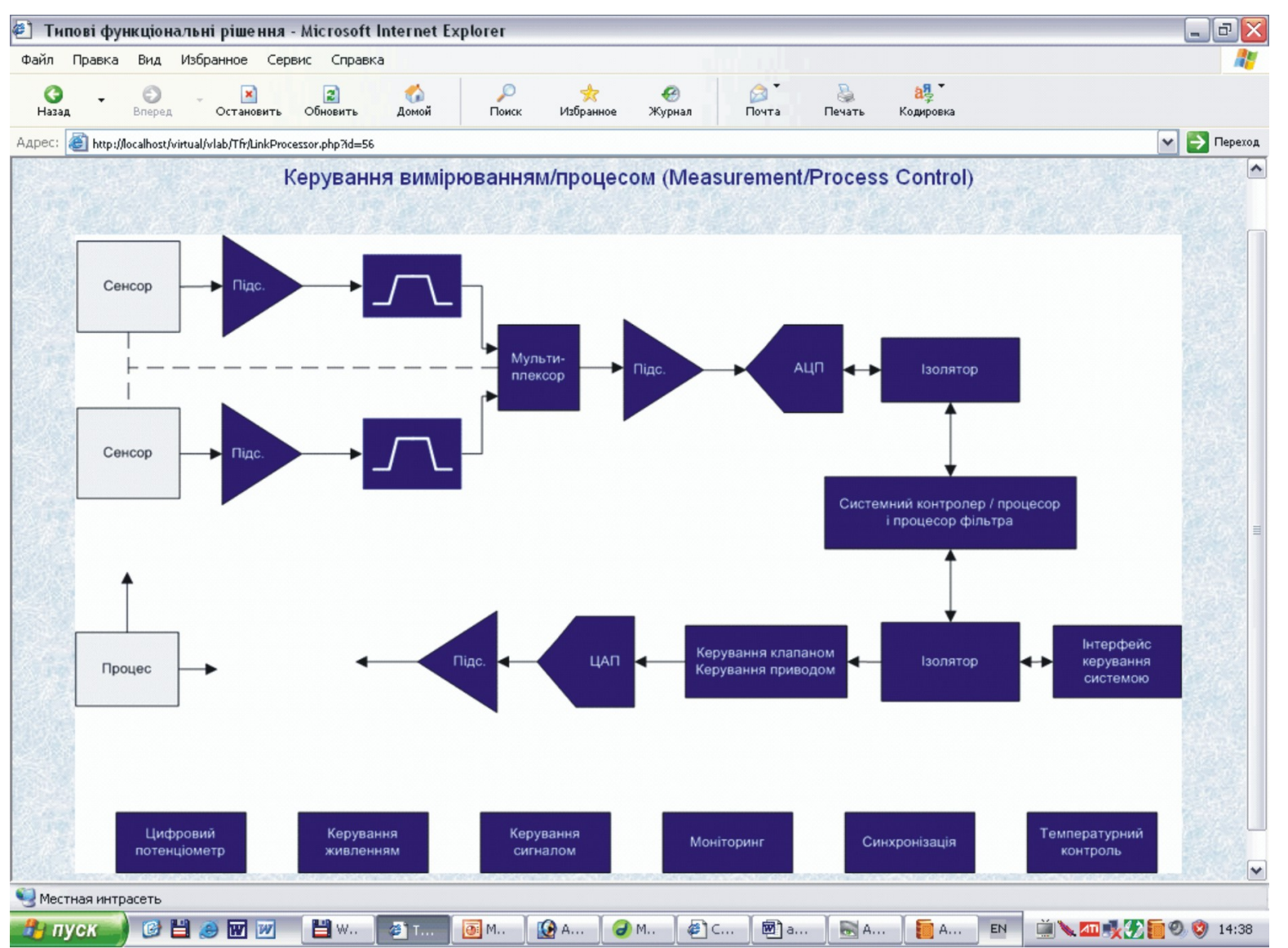

Fig. 3 - Example of typical functional solution

Practical using of reliability calculation is shown below, as example, for: 1) one microelectronic component; 2) whole device with different amount of components.

Calculations were made for microelectronic components of Analog Devices, Inc. All necessary data were taken from company official site http://www.analog.com. Methods and formulas for calculation are described in [6]. Now in the VLCAD library there are data about reliability attributes for almost 1000 microelectronic components.

Reliability of microelectronic components is estimated by following characteristics: 1) failure rate $\lambda$, which is measured in FIT (failure in $10^{9}$ component hours); 2) mean time to failure $\mathrm{T}_{0}$ (in hours (h.) and years).

Reliability characteristics were calculated for following $60 \%$ i $90 \%$ confidence levels (CL).

Calculation of reliability characteristics of separate microelectronic component. For calculation we choose 14-bits DAC AD7840 (Analog Devices), which is made by means of "CMOS $>2,0 \mu \mathrm{m}$ " technology. On the site you can find following reliability parameters for this component accordingly to manufacture technology:

$\mathrm{CL}=60 \%: \quad \lambda=3 \cdot 10^{-9} \mathrm{~h} ., \quad \mathrm{T}_{0}=337841942 \mathrm{~h}$. (38566 years);

$$
\mathrm{CL}=90 \%: \quad \lambda=7 \cdot 10^{-9} \mathrm{~h} ., \quad \mathrm{T}_{0}=134441015 \mathrm{~h} .
$$
(15347 years);

Having made calculations we get the following data:

exponential distribution law:

$\mathrm{CL}=60 \%: \quad \lambda=2,96 \cdot 10^{-9} \mathrm{~h} ., \quad \mathrm{T}_{0}=337764531 \mathrm{~h}$. (38558 years);

ДГ $=90 \%: \quad \lambda=7,43 \cdot 10^{-9} \mathrm{~h} ., \quad \mathrm{T}_{0}=134591823 \mathrm{~h}$. (15364 years);

DN-distribution law:

$\mathrm{CL}=60 \%: \quad \lambda=2,96 \cdot 10^{-9} \mathrm{~h} ., \quad \mathrm{T}_{0}=1704200 \mathrm{~h}$. (195 years);

$\mathrm{CL}=90 \%: \quad \lambda=7,43 \cdot 10^{-9} \mathrm{~h} ., \quad \mathrm{T}_{0}=1534950 \mathrm{~h}$. (175 years).

For calculations, which placed above, there were used integrated data for manufacture technology, which can be found on the official site.

The results show, that calculations, which are made by means of exponential distribution law, are equal to parameters, which are on the site of manufacturer. It is so, because company Analog Devices uses method on the base of exponential distribution law of failure probability [7]. Results, which calculated by means of using DN-distribution law, are different from given by manufacturer (exponential distribution law). This difference in the results is prospective [5]. Exponential model overrates real reliability characteristics of separate 


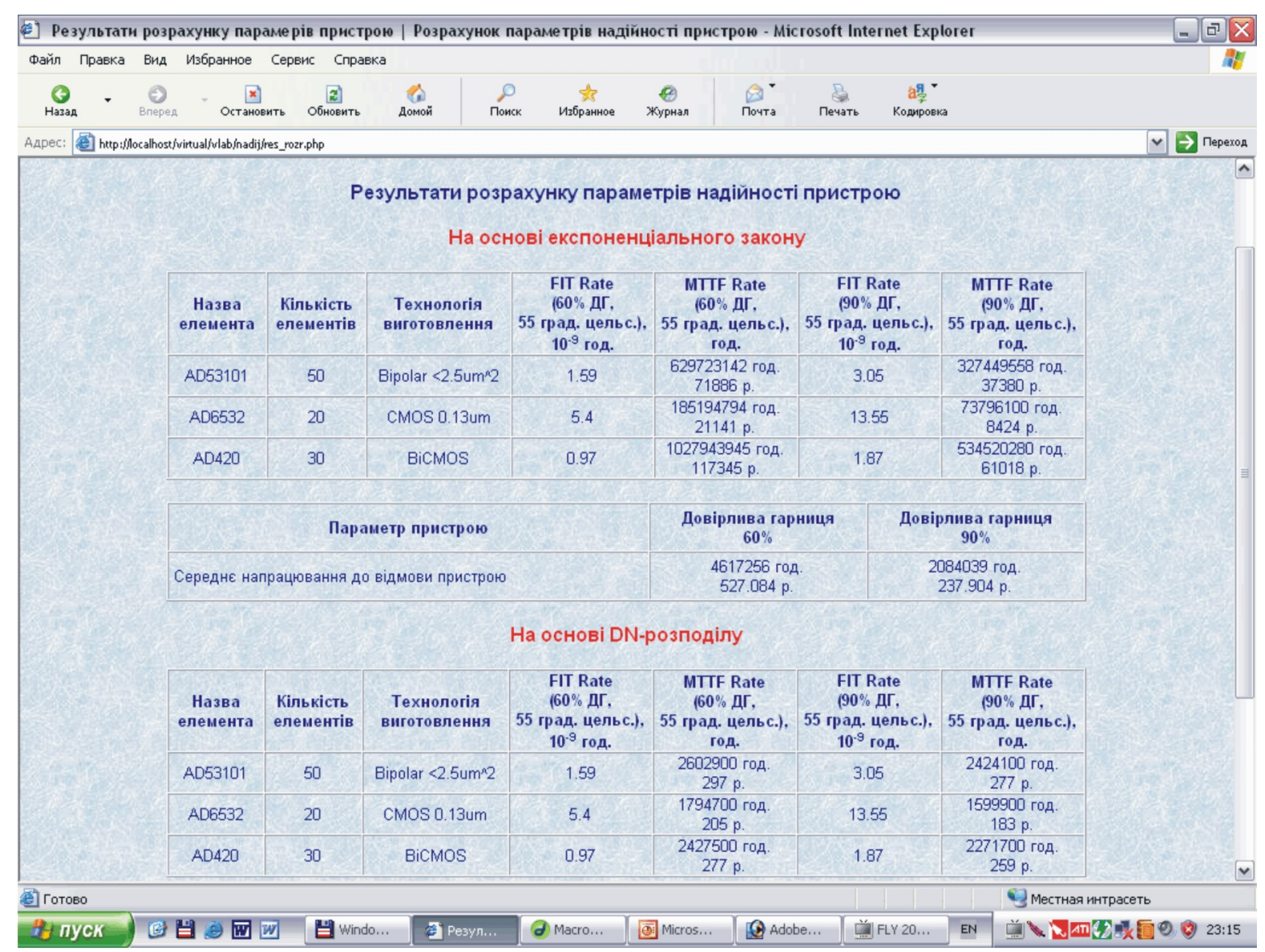

Fig. 4 - Results of calculations of reliability parameters of whole device

microelectronic component.

Calculation of reliability characteristics of whole device with different amount of microelectronic components. For calculation of reliability characteristics of whole device as input data there were used results of calculation of separate component reliability characteristics. As components of device we choose DAC AD7840 and operational amplifier OP497 ("CMOS $>2,0 \mu \mathrm{m} "$ and "Bipolar $>2,5 \mu \mathrm{m}^{2}$ accordingly").

For whole device unit calculate only mean time to failure $T_{0}$ (in hours and years) by means of two methods.

The results of calculations of reliability characteristics of whole device for 3 cases are shown in table 1. In the first case the total amount of microelectronic components in the device is equal
10 components (5 AD7840 and 5 OP497), in the second case - 1000 components (500 AD7840 and 500 OP497) and in the third case - 110 thousand components (55 thousand AD7840 and 55 thousand OP497).

As in the case of separate component the calculations show, that reliability characteristics, which are calculated by means of using of exponential distribution law, are overrated. This statement is actual for devices with small amount of microelectronic components. When the amount of components in device is enough large the reliability characteristics, which are calculated by means of using of exponential distribution law, are underrated in comparison with characteristics, which are calculated by means of using of DN-distribution law of fault probability.

Table 1. Result of calculations of reliability characteristics of whole device

\begin{tabular}{|l|c|c|c|c|c|}
\hline \multirow{2}{*}{$\begin{array}{c}\text { Amount of microelectronic } \\
\text { components }\end{array}$} & \multirow{2}{*}{$\mathrm{T}_{0}$} & \multicolumn{2}{|c|}{$\begin{array}{c}\text { Exponential distribution law } \\
\text { of fault probability }\end{array}$} & \multicolumn{2}{c|}{$\begin{array}{c}\text { DN-distribution law } \\
\text { of fault probability }\end{array}$} \\
\cline { 3 - 6 } & & $\mathrm{CL}=60 \%$ & $\mathrm{CL}=90 \%$ & $\mathrm{CL}=60 \%$ & $\mathrm{CL}=90 \%$ \\
\hline $\mathbf{1 0}$ & hours & 54802369 & 21837553 & 645130 & 583133 \\
(5 of each) & years & 6255,978 & 2492,871 & 73,645 & 66,568 \\
\hline $\mathbf{1 0 0 0}$ & hours & 548024 & 218376 & 64513 & 58313 \\
(500 of each) & years & 62,56 & 24,929 & 7,364 & 6,657 \\
\hline $\mathbf{1 1 0 ~ 0 0 0}$ & hours & 4982 & 1985 & 6151 & 5560 \\
(55 thousand of each) & years & 0,569 & 0,227 & 0,702 & 0,635 \\
\hline
\end{tabular}




\section{VLCAD IN EDUCATION, TRAINING AND SCIENTIFIC RESEARCHES}

As was written above, one of VLCAD advantages is possibility of using VLCADs for training future specialists and in scientific researches. For efficient using in education virtual laboratory has next facilities:

1) databases with large amount of information about microelectronic components and their parameters, typical functional solutions, components model, evaluating boards etc;

2) special calculation units (for example, for calculation of reliability parameters of microelectronic components and whole devices etc), and also theoretical materials with detailed explaining of calculation methods;

3) possibility of using of calculation units independently each from other;

4) databases with magazines, articles and other publications in electronic form and search subsystem for them;

5) databases of links on specialized sites (magazines, companies, forums etc) in Internet;

6) possibility of access to databases and other materials of VLCAD from Internet, for example, from computer class of educational university;

7) possibility to involve students to the real projects, which are in VLCAD databases. This gives opportunity to select students, who have gift of fast and optimal solving tasks in the field of computer devices designing;

8) possibility to fill databases with problem situations, which have place in real projects, for future using in education and training.

For effective using during scientific researches virtual laboratory has large databases of specialized information and calculation units, which allow saving scientists and developers from complicated and routine calculations and searches of information. Opportunity of access to VLCAD from Internet allows having this tool always at hand from any place of world.

Virtual laboratory has database of links to interactive tools, which enough help during designing and developing of electronic devices. Mainly such interactive tools are presented at sites of manufacturers of microelectronic devices and software, and mainly are intended for performing of one complicated or simple operation (for example, for calculation of electronic switch parameters, signal setting time etc). For lighten work with these interactive tools the data manuals for work with these tools and algorithms of operation are presented in VLCAD.

\section{CONCLUSIONS}

Structure and organization of typical virtual laboratory for computer-aided design of biosensors ware considered in the article. The goal of creating of virtual laboratory is to lighten work of design engineers and to make possible for scientists and specialists of different areas to develop their own devices, estimate work hypothesis and fulfill virtual experimental researches.

VLCAD lets to analyze the functions of separated device units and to evaluate the work of whole device without using complex equipment and expensive actual tests. Also it lets to reduce both cost and time of design, and that is very important today.

VLCAD has next facilities, which give possibility for effective using of the virtual laboratory during education and science investigations: presence of large databases with information about microelectronic components and their characteristics; using of different procedures for calculation of characteristics of components or whole devices; presence of descriptions of calculation procedures; presence of a large amount of electronic publications and references to Internet resources; possibility of access to virtual laboratory from Internet and local network of organization.

\section{REFERENCES}

[1] O. Palagin, A. Kurgaev. Problem orientation in the development computer arhitecture. Cibernetics and system analisys 4 (2003). p. 167-180 (in Russian)

[2] O. Palagin, Yu. Brajko, I. Galelyuka, R. Imamutdinova, V. Romanov. Structure organization of virtual laboratory for design of computer aides. Computer facilities, networks and systems 4 (2005), pp. 47-56 (in Russian).

[3] O. Palagin, V. Romanov, I. Galelyuka. Virtual boards and their application for designing of biosensor devices. Proceedings of 12th International conference "Automation-2005", Harkiv, Ukraine 30 May - 3 June 2005, p. 14.

[4] O. Palagin, V. Romanov, I. Galelyuka. Estimating of reliability during virtual design of computer devices. Radioelectronic and computer systems, 2007, № 7(26), pp. 215-218.

[5] V. Streljnikov. Estimating of resource of electronic technique products. Mathematical machines and systems, 2004, № 2, pp. 186-195.

[6] O. Palagin, V. Romanov, I. Galelyuka. Virtual methods of designing of complicated systems: estimation of reliability. Information technology 
and computer engineering, 2005, № 3, pp. 147150.

[7] ADI reliability handbook. Analog Devices, Inc., 2004.

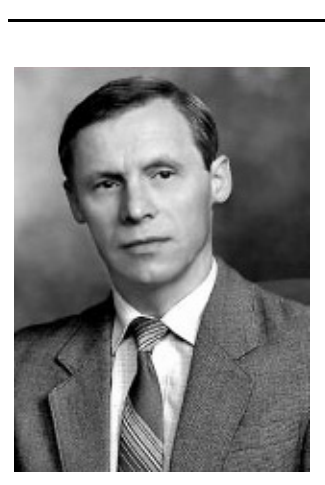

\section{Palagin}

academician of NAS of Ukraine, doctor of technical science, professor, deputy director of V.M. Glushkov Institute of Cybernetics of National Academy of Sciences of Ukraine, Computer Sciences.

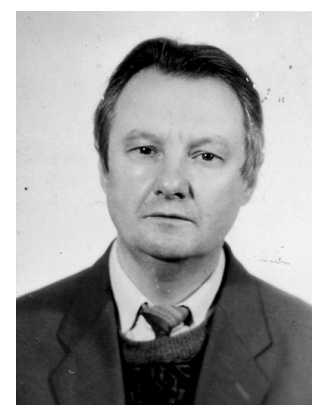

Romanov Volodymyr, doctor of technical science, professor, head of department of V.M. Glushkov Institute of Cybernetics of National Academy of Sciences of Ukraine, Computer Sciences.

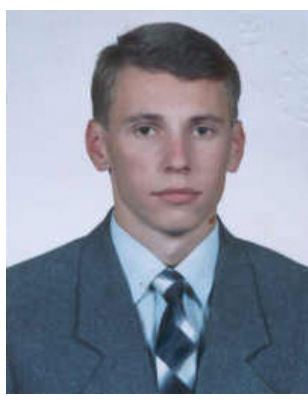

Galelyuka lgor, science fellow of V.M. Glushkov Institute of Cybernetics of National Academy of Sciences of Ukraine, Computer Sciences.

Kachanovska Maryna, programming engineer of V.M. Glushkov Institute of Cybernetics of National Academy of Sciences of Ukraine, Computer Sciences. 